University of New Orleans

ScholarWorks@UNO

2012

\title{
Dynamics and Collective State of Ordered Magnetic Nanoparticles in Mesoporous Systems
}

Jose M. Vargas

University if New Orleans, jmvargas@uno.edu

Abhishek Srivastava

University of New Orleans, abhi.alld@gmail.com

Ezra Garza

University of New Orleans, epgarza@my.uno.edu

Gabriel Caruntu

University of New Orleans, gcaruntu@uno.edu

Amin Yourdkhani

University of New Orleans, ayourdkh@uno.edu

See next page for additional authors

Follow this and additional works at: https://scholarworks.uno.edu/chem_facpubs

Part of the Chemistry Commons

\section{Recommended Citation}

J. Appl. Phys. 112, 094309 (2012)

This Article is brought to you for free and open access by the Department of Chemistry at ScholarWorks@UNO. It has been accepted for inclusion in Chemistry Faculty Publications by an authorized administrator of ScholarWorks@UNO. For more information, please contact scholarworks@uno.edu. 
Authors

Jose M. Vargas, Abhishek Srivastava, Ezra Garza, Gabriel Caruntu, Amin Yourdkhani, and Leonard Spinu 
Magnetic properties of variable-sized $\mathrm{Fe}_{3} \mathrm{O}_{4}$ nanoparticles synthesized from non-aqueous homogeneous solutions of polyols

This article has been downloaded from IOPscience. Please scroll down to see the full text article.

2007 J. Phys. D: Appl. Phys. 405801

(http://iopscience.iop.org/0022-3727/40/19/001)

View the table of contents for this issue, or go to the journal homepage for more

Download details:

IP Address: 137.30.164.204

The article was downloaded on 05/03/2013 at 20:24

Please note that terms and conditions apply. 


\title{
Magnetic properties of variable-sized $\mathrm{Fe}_{3} \mathrm{O}_{4}$ nanoparticles synthesized from non-aqueous homogeneous solutions of polyols
}

\author{
Daniela Caruntu, Gabriel Caruntu and Charles J O'Connor \\ Advanced Materials Research Institute, University of New Orleans, New Orleans, \\ LA 70148, USA \\ E-mail: dcarunt1@uno.edu
}

Received 26 April 2007, in final form 22 July 2007

Published 21 September 2007

Online at stacks.iop.org/JPhysD/40/5801

\begin{abstract}
The magnetic behaviour of well-dispersed monodisperse $\mathrm{Fe}_{3} \mathrm{O}_{4}$ nanoparticles with sizes varying between 6.6 and $17.8 \mathrm{~nm}$ prepared in a non-aqueous medium was investigated. The smaller nanocrystals exhibit superparamagnetism with the blocking temperatures increasing with the particle size, whereas the biggest particles are ferromagnetic at room temperature. The saturation magnetization values are slightly smaller than that of the bulk material, suggesting the existence of a disordered spin configuration on their surface. The thickness of the magnetically inert shell was estimated from the size variation of the magnetization at $1.9 \AA$. The dipole-dipole interactions between the particles were tuned by changing the interparticle distances, e.g. by diluting the nanopowders in a non-magnetic matrix at concentrations ranging from 0.25 to $100 \mathrm{wt} \%$. As the strength of the interactions is decreased with dilution, the energy barrier is substantially lowered; this will induce a drastic decrease of both the blocking temperatures and the coercivity with decreasing concentration of the nanoparticles.
\end{abstract}

(Some figures in this article are in colour only in the electronic version)

\section{Introduction}

Transition metal ferrites, $\mathrm{M}^{\mathrm{II}} \mathrm{Fe}_{2}^{\mathrm{III}} \mathrm{O}_{4}$, are magnetic materials with a cubic spinel-type structure which have been extensively used in various technological applications in the past decades $[1,2]$. Owing to their easy preparation, low fabrication cost, high chemical stability and unique electrical, optical, thermal, rheological, catalytic and magnetic properties, ferrites, either in the form of nanopowders or surface-stabilized nanoparticles suspended in a carrier liquid (ferrofluid) [3], have widespread use in electronics [4], magneto-optics [5], magnetocaloric refrigeration [6], dynamic sealing [7], high-density information storage [8], oscillation damping [9] and catalysis [10]. Among the nanocrystalline ferrites, $\mathrm{Fe}_{3} \mathrm{O}_{4}$ and $\gamma-\mathrm{Fe}_{2} \mathrm{O}_{3}$ have attracted particular interest as ideal candidates for different biomedical applications including enzyme encapsulation [11], biosensor design [12, 13], cell labelling/separation [14] and oligonucleotide identification [15], magnetic resonance imaging (MRI) [16-18], tumour hyperthermia [19, 20] and magneticallytargeted drug delivery [21-24] due to their dimensions being comparable to the cells and biomolecules, low toxicity and biocompatibility, high saturation magnetization values and their easy manipulation with low magnetic fields.

As the size of the particles decreases below $100 \mathrm{~nm}$, a large fraction of the constituting atoms are found on the surface of the nanocrystals; this induces significant changes in the magnetic structure and properties of the nanophase materials as compared with their bulk counterparts. Specifically, the domain wall structure encountered in the bulk crystalline ferrites is replaced by a single domain structure characteristic of each particle, thus leading to new phenomena, such as 
superparamagnetism [25], extra anisotropy contributions [26] and spin canting (disordered spin configuration) [27, 28]. Superparamagnetism refers to the random fluctuation of the magnetization of the single-domain particles when the thermal energy overcomes the anisotropy energy barrier. At low temperatures the magnetization of each nanoparticle is oriented parallel to a particular crystallographic direction called the easy axis along which the energy of the particle is minimized. The magnetization of the nanoparticle remains blocked in this minimum energy position until an amount of energy at least equal to the anisotropy of the particle is provided to the system. Increasing the temperature above a certain value, known as the blocking temperature $\left(T_{\mathrm{B}}\right)$, the thermal energy will overcome the anisotropy energy $\left(E_{\mathrm{A}}\right)$ and the magnetization of each particle begins to fluctuate between the two directions of the easy axes. Under these conditions, the magnetic system behaves as a classical paramagnet [29]. According to StonerWohlfarth theory, the blocking temperature is higher as the volume of the nanoparticles increases:

$$
T_{\mathrm{B}}=\frac{K}{25 k_{\mathrm{B}}} \cdot V
$$

where $K$ represents the anisotropy constant and $k_{\mathrm{B}}$ is the Boltzmann constant [30]. In general, the magnetic behaviour of the nanoparticles is the result of the interplay between the bulk magnetocrystalline characteristics, finite size effects and collective magnetostatic interactions between the particles. However, the delimitation of each individual contribution to the total magnetization of the magnetic system would require not only the preparation of well-dispersed nanocrystals with controllable sizes and shapes but also a strict control over the interactions between them. Finite size effects include the single-domain magnetic structure and the surface spin disorder and originate from the high surface/volume ratio of the small particles, whereas the interparticle interactions refer to the exchange and dipole-dipole interactions. The study of the interparticle interactions is not trivial since they can change significantly the magnetic response of a nanosized system by increasing the anisotropy energy barrier $\left(E_{\mathrm{A}}\right)$ necessary to overcome the blocking of the fine particles. Herein, we report on the influence of the finite size effects and the interparticle interactions on the magnetic properties of the oleate-capped $\mathrm{Fe}_{3} \mathrm{O}_{4}$ nanocrystals synthesized in nonaqueous homogeneous solutions of polyols (diethylene glycol and $\mathrm{N}$-methyl diethanolamine). The experiments were performed on two series of samples, one containing $\mathrm{Fe}_{3} \mathrm{O}_{4}$ particles with various sizes ranging from 6.6 to $17.8 \mathrm{~nm}$ and the other containing $6.6 \mathrm{~nm}$ particles diluted at different concentrations of paraffin.

\section{Experimental}

\subsection{Chemicals}

Iron(III) chloride hexahydrate $97 \%$, toluene $99.5 \%$, methyl alcohol $99.8 \%$ and hexanes $98.5 \%$ were purchased from Merck. Diethylene glycol 99\%, N-methyl diethanolamine $99+\%$, sodium hydroxide $97 \%$ (20-40 mesh beads), ethyl acetate $99.5 \%$ and decane $99+\%$ were obtained from Aldrich. Iron(II) chloride tetrahydrate $99 \%$ was purchased from Alfa
Aesar, oleic acid 90\% from Fisher and absolute ethyl alcohol from AAper Alcohol and Chemical Co. Chemicals and solvents were used without further purification. The airsensitive chemicals were manipulated in a VAC glovebox with a nitrogen atmosphere.

\subsection{Synthesis}

The synthetic procedures for the preparation of variably sized oleate-capped $\mathrm{Fe}_{3} \mathrm{O}_{4}$ nanocrystals were described previously in detail in [31-32]. For example, the $\sim 6 \mathrm{~nm}$ sized magnetite nanoparticles were prepared in diethylene glycol (DEG) solutions obtained by dissolving a mixture of $2 \mathrm{mmol} \mathrm{FeCl}_{2} \cdot 4 \mathrm{H}_{2} \mathrm{O}$ and $4 \mathrm{mmol} \mathrm{FeCl}_{3} \cdot 6 \mathrm{H}_{2} \mathrm{O}$ in a $80 \mathrm{~g}$ solvent in a Schlenk flask under protection with argon. Separately, $16 \mathrm{mmol}$ of $\mathrm{NaOH}$ was dissolved in $40 \mathrm{~g}$ diethylene glycol. The solution of $\mathrm{NaOH}$ was added to the solution of metal chlorides with stirring at room temperature causing an immediate colour change from yellow-brown to deep greenbrown. After $3 \mathrm{~h}$, the temperature of the solution was raised for $1.5 \mathrm{~h}$ to $210^{\circ} \mathrm{C}$ and then kept constant for $2 \mathrm{~h}$ in the temperature range $210-220^{\circ} \mathrm{C}$. In order to obtain magnetite nanoparticles soluble in non-polar solvents, a diethylene glycol solution of oleic acid ( $2.6 \mathrm{mmol}$ oleic acid per $20 \mathrm{~g}$ DEG) was added to the reaction mixture at a high temperature. This addition immediately precipitated the solids. The mixture was cooled to room temperature and then centrifuged. The precipitates were washed with methanol and dissolved in $20 \mathrm{ml}$ toluene. The resulting solution was centrifuged and mixed with 2-3 volumes of methanol. The precipitate was separated by centrifuging, washed with methanol and kept either moistened with methanol or dispersed in non-polar solvents (hexanes, toluene and decane). When magnetic measurements were planned, the solids were dried in a flow of nitrogen.

By analogy, largest magnetite nanoparticles $(\sim 17 \mathrm{~nm})$ were prepared following a similar synthetic procedure but using $\mathrm{N}$-methyl diethanolamine (NMDEA) instead of diethylene glycol (DEG), whereas the $11 \mathrm{~nm}$ sized magnetite nanoparticles were obtained in a mixture of diethylene glycol (DEG) and N-methyl diethanolamine (NMDEA) $(1: 1, \mathrm{w} / \mathrm{w})$ heated for $3 \mathrm{~h}$ in the temperature interval $210-220^{\circ} \mathrm{C}$. In all the cases the resultant black solid was dried in a flow of nitrogen.

\subsection{Characterization}

The morphology of the magnetite nanoparticles was examined with a JEOL JEM 2010 transmission electron microscope (TEM) at $200 \mathrm{KV}$. TEM samples were prepared by a conventional technique consisting of pouring several drops of the ferrofluid solution onto carbon coated $\mathrm{Cu}$ grids followed by the slow evaporation of the solvent. Thermogravimetric analyses (TGA) were carried out in argon at a heating rate of $2^{\circ} \mathrm{C} \mathrm{min}^{-1}$ up to $600{ }^{\circ} \mathrm{C}$ using a SDTQ 600 analyzer. The magnetic measurements of both as-prepared $\mathrm{Fe}_{3} \mathrm{O}_{4}$ nanoparticles and samples diluted in paraffin were performed with a Superconducting Quantum Interference Device (SQUID) in the temperature range $5-300 \mathrm{~K}$ and magnetic fields up to $5 \mathrm{~T}$. The zero-field-cooled (ZFC) curves were obtained by cooling the magnetite samples from 300 to $5 \mathrm{~K}$ in the absence of an external magnetic field, followed by the measurement 

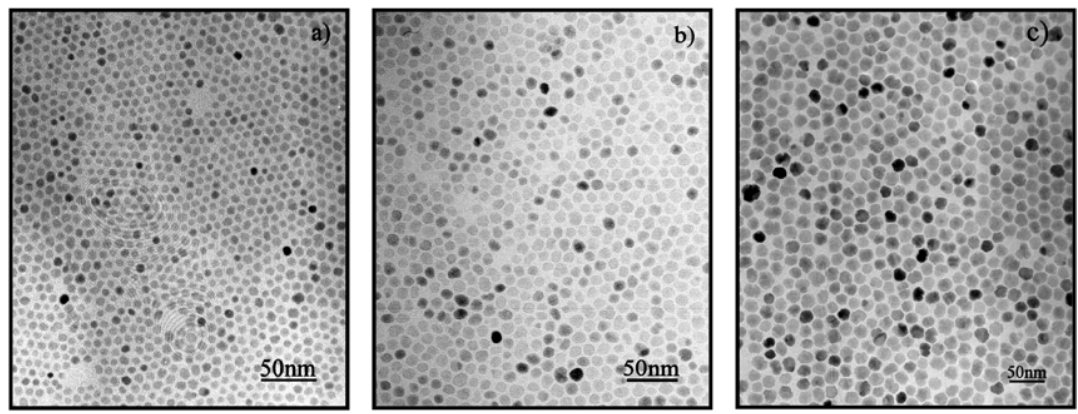

Figure 1. Representative TEM images of oleate-capped magnetite nanoparticles prepared from neat DEG (a), a mixture of DEG and $\operatorname{NMDEA}(1: 1, \mathrm{w} / \mathrm{w})(b)$ and neat NMDEA $(c)$.

of the magnetization under a magnetic field of $100 \mathrm{Oe}$ as the temperature was raised back to room temperature. The field-cooled (FC) measurements were carried out in a similar way, except for the cooling process which was performed under an external magnetic field of $100 \mathrm{Oe}$. The hysteresis loop measurements were performed at different temperatures ranging from 5 to $300 \mathrm{~K}$ under magnetic fields of up to $5 \mathrm{~T}$.

\section{Results and discussion}

Highly crystalline magnetite $\left(\mathrm{Fe}_{3} \mathrm{O}_{4}\right)$ nanoparticles were prepared at elevated temperatures by the hydrolysis of chelate iron alkoxide complexes in solutions of corresponding polyols, diethylene glycol (DEG) and N-methyl diethanolamine (NMDEA). The fact that $\mathrm{Fe}_{3} \mathrm{O}_{4}$ was obtained instead of the structurally related $\gamma-\mathrm{Fe}_{2} \mathrm{O}_{3}$ was confirmed by a series of controlled oxidation experiments in tandem with powder $\mathrm{x}$-ray diffraction as previously documented. Polyols play a multiple role in the formation of nanocrystalline $\mathrm{Fe}_{3} \mathrm{O}_{4}$ particles serving not only as solvents and complexing agents for the iron (II, III) precursors but also as stabilizing agents for the resulting nanocrystals. Moreover, the presence of monolayers of polyol molecules adsorbed on the nanocrystals' surface induces their solubility in polar solvents such as water, methanol and ethanol. The polyol molecules can be exchanged at elevated temperatures for long-chain carboxylic acids which bind covalently to the surface iron ions via carboxylate groups, thereby stabilizing the particles against agglomeration and rendering them soluble in non-polar media (toluene, hexanes and decane) $[31,32]$. These fatty acids are also essential in minimizing the exchange interactions between the superficial iron ions of neighbouring $\mathrm{Fe}_{3} \mathrm{O}_{4}$ nanocrystals such that the interparticle interactions are dominated by the dipole-dipole ones [33,34].

Adjusting the complexing strength of the reaction medium by using either neat solvents (DEG or NMDEA) or a $1: 1$ $(\mathrm{w} / \mathrm{w})$ mixture of the two polyols allowed tuning of the particle dimensions in the range $6-17 \mathrm{~nm}$ [32]. Figure 1 shows the TEM images of the $\mathrm{Fe}_{3} \mathrm{O}_{4}$ nanoparticles prepared from neat DEG (a), neat NMDEA (c) and a mixture of DEG and $\operatorname{NMDEA}(1: 1, w / w)(b)$, respectively. As revealed by the TEM micrographs, the $\mathrm{Fe}_{3} \mathrm{O}_{4}$ nanoparticles obtained from neat DEG and the mixture of the two polyols have spheroidal shapes, whereas those synthesized from neat NMDEA are faceted. The nanocrystals are individual and relatively uniform-sized.
They arrange in monolayers which exhibit a short range order due to the presence of small fractions of tiny particles and shapeless aggregates (figures $1(b)$ and $(c)$ ) indicating that the $\mathrm{Fe}_{3} \mathrm{O}_{4}$ nanoparticles are formed through the aggregation of 'primary particles' [32]. The average particle sizes and the size distributions were estimated by counting 150 nanoparticles.

As anticipated, the nature of the reaction medium significantly influences the size of the resulting nanocrystalline particles: while the DEG-mediated reaction leads to $6.6 \mathrm{~nm}$ sized $\mathrm{Fe}_{3} \mathrm{O}_{4}$ particles (standard deviation, $\sigma=11.3 \%$ ), a mixture of DEG and NMDEA $(1: 1, \mathrm{w} / \mathrm{w})$ results in the formation of particles with an average diameter of $11.6 \mathrm{~nm}$ $(\sigma=9.4 \%)$ which can be increased to $17.8 \mathrm{~nm}(\sigma=10.5 \%)$ when the reaction is performed in neat NMDEA (figure 2). The crystallite sizes calculated from the corresponding x-ray diffraction patterns by using Scherrer's formula led to values similar to those obtained from the TEM measurements, suggesting that the nanopowders consist of single crystalline particles. Additionally, the XRD patterns showed that nanopowders are single phase materials that crystallize into cubic-type structures with the cell parameters comparable to that of the crystalline bulk magnetite $(a=8.396 \AA)$ [32].

Figure 3 illustrates the temperature $(T)$ dependence of the ZFC and FC magnetizations $(M)$ measured for variablesized $\mathrm{Fe}_{3} \mathrm{O}_{4}$ nanoparticles coated with oleate ligand. For the nanoparticles prepared from neat DEG (figure 3(a)) and those synthesized from a $1: 1$ mixture of DEG and NMDEA (figure 3(b)), the ZFC and FC curves, which coincide initially, start to separate and follow different trends as the temperature is decreased from 300 to $5 \mathrm{~K}$. In the FC mode, the magnetization either increases slightly and then levels off (figure $3(a)$ ) or decreases to eventually reach a plateau (figure $3(b)$ ), whereas the ZFC magnetization shows a maximum followed by a steady decrease to a value approaching zero in the low temperature region. The shape of the FC curves in the figures $3(a)$ and (b) is the result of the presence of dipole-dipole interactions between the oleate-capped particles $[35,36]$. Moreover, the variation of the magnetization in the $\mathrm{ZFC}$ and $\mathrm{FC}$ modes indicates a superparamagnetic behaviour for the 6.6 and $11.6 \mathrm{~nm}$ sized $\mathrm{Fe}_{3} \mathrm{O}_{4}$ particles. The maximum in the $\mathrm{ZFC}$ curve defines the blocking temperature $T_{\mathrm{B}}$, where the thermal energy becomes comparable to the anisotropy energy barrier $\left(E_{\mathrm{A}}\right)$. For temperatures below $T_{\mathrm{B}}$, the magnetization of each nanoparticle aligns with the direction of the easy axis and cannot be further changed due to the existence of the anisotropy energy barriers. Owing to the random orientation of the easy 

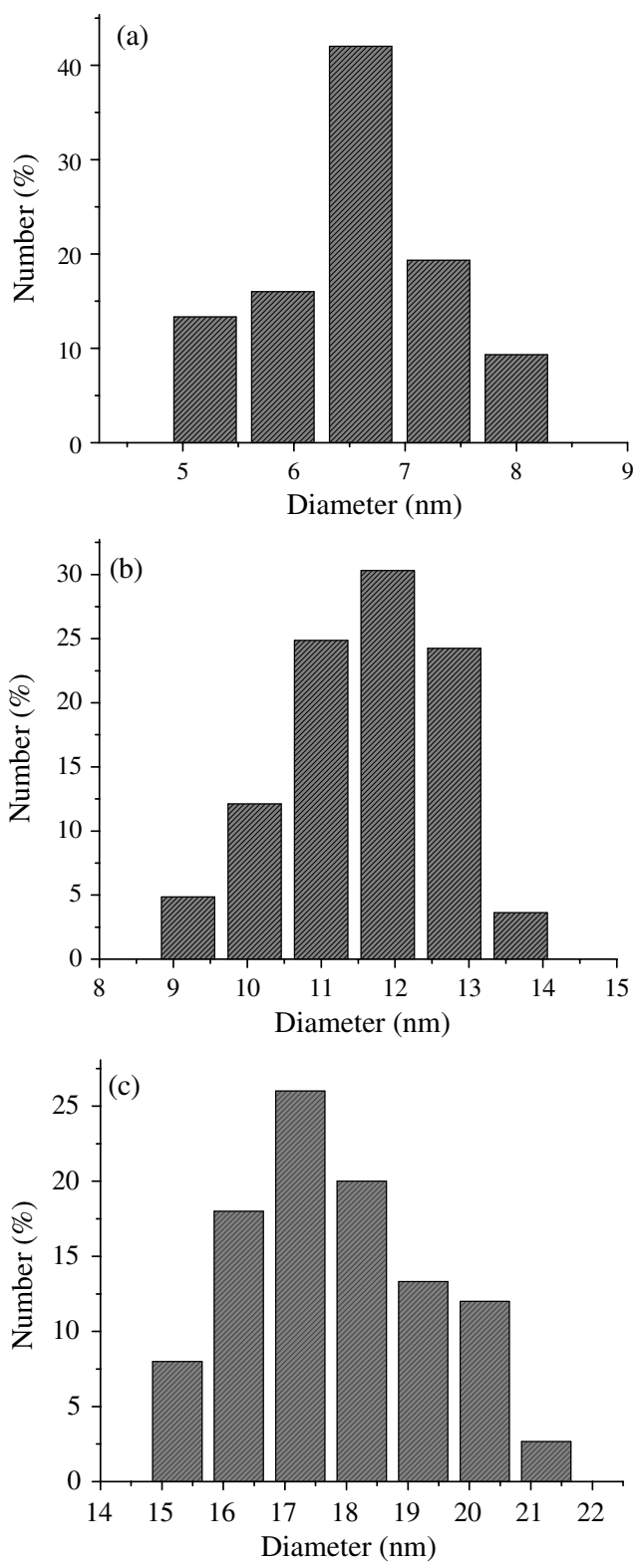

Figure 2. Histograms of size distribution for magnetite nanocrystals synthesized from neat DEG $(a)$, a mixture of DEG and NMDEA $(1: 1, \mathrm{w} / \mathrm{w})(b)$ and neat NMDEA $(c)$.

axes of the nanoparticles, the total magnetization approaches zero at low temperatures. However, when the sample is progressively warmed up to $300 \mathrm{~K}$, an increasing number of particles will acquire a thermal energy comparable to the anisotropy energy, thus switching their magnetizations from the easy axes to the direction of the magnetic field. This leads to a progressive increase in the magnetization of the sample. At temperatures higher than $T_{\mathrm{B}}$, the magnetization of each particle begins to fluctuate between the two directions of the easy axis (superparamagnetic relaxation). The values of the blocking temperature were found to vary between $203 \mathrm{~K}$ for the particles obtained from neat DEG $(6.6 \mathrm{~nm})$ to $264 \mathrm{~K}$ for those prepared from a $1: 1$ mixture of DEG and NMDEA $(11.6 \mathrm{~nm})$. In the case of particles synthesized from neat NMDEA $(17.8 \mathrm{~nm})$, no maximum was observed in the $\mathrm{ZFC}$ curve. However, since the ZFC curve presents a shoulder in the high temperature
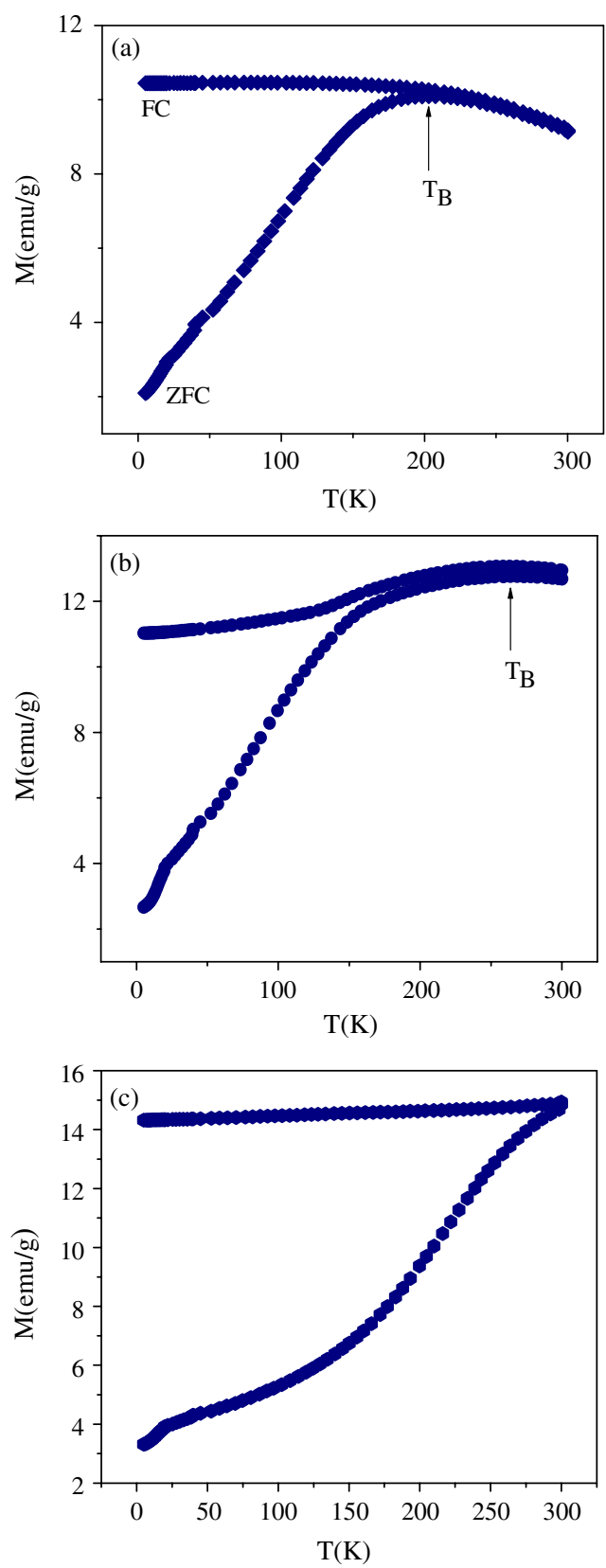

Figure 3. Magnetization $(M)$ versus temperature $(T)$ measured in both the ZFC and the FC modes for oleate-capped magnetite nanocrystals with various sizes: $6.6 \mathrm{~nm}(a), 11.6 \mathrm{~nm}(b)$ and $17.8 \mathrm{~nm}(c)$, respectively.

region, we assume that the blocking temperature of the biggest nanocrystals is situated above $300 \mathrm{~K}$. The observed variation of the blocking temperature with the average size of the $\mathrm{Fe}_{3} \mathrm{O}_{4}$ nanocrystals is consistent with Stoner-Wohlfarth theory which predicts an increase of the anisotropy energy barrier $\left(E_{\mathrm{A}}\right)$ and, consequently, an increase of the blocking temperature $\left(T_{\mathrm{B}}\right)$ as the volume of the nanoparticles increases.

Assuming that the particles are non-interacting and possess a uniaxial anisotropy, the corresponding blocking temperatures $\left(T_{\mathrm{B}}\right)$ can be used to estimate the values of the anisotropy energy constant $(K)$ of the oleate-capped $\mathrm{Fe}_{3} \mathrm{O}_{4}$ nanocrystals. The calculated values of the constant are $K=4.74 \times 10^{5} \mathrm{~J} \mathrm{~m}^{-3}$ for the particles with an average 
diameter of $6.6 \mathrm{~nm}$ and $K=1.11 \times 10^{5} \mathrm{~J} \mathrm{~m}^{-3}$ for those with a mean size of $11.6 \mathrm{~nm}$, respectively. These values are about one order of magnitude higher than that of the bulk material $\left(K_{\text {(bulk) }}=0.135 \times 10^{5} \mathrm{~J} \mathrm{~m}^{-3}\right.$ ) [28] and were found to increase with decreasing size of the nanoparticles. Additionally, they are slightly larger, but yet in good agreement with the $K$ values estimated by Mössbauer spectroscopy for $6 \mathrm{~nm}(K=1.4 \times$ $\left.10^{5} \mathrm{~J} \mathrm{~m}^{-3}\right)$ and $12 \mathrm{~nm}\left(K=0.9 \times 10^{5} \mathrm{~J} \mathrm{~m}^{-3}\right)[37,38]$ particles or by using the equation $K V=25 k_{\mathrm{B}} T$ for $7 \mathrm{~nm} \mathrm{Fe}_{3} \mathrm{O}_{4}$ particles $\left(K=2.8 \times 10^{5} \mathrm{~J} \mathrm{~m}^{-3}\right)$ [26], but much lower than that corresponding to $3-7 \mathrm{~nm}$ sized particles obtained by the waterin-oil microemulsion technique $\left(K=10^{6} \mathrm{~J} \mathrm{~m}^{-3}\right)$ [39]. The anisotropy constant $(K)$ of the nanocrystalline materials can incorporate different contributions from magnetocrystalline shape and surface anisotropy $[26,40]$. Thus, assuming that the magnetocrystalline anisotropy of the $\mathrm{Fe}_{3} \mathrm{O}_{4}$ nanoparticles prepared in the polyol media is invariant to the reaction conditions (the nature of the solvent, the heating time) and the shape of the nanoparticles does not change significantly with their size, the variation trend in $K$ can be ascribed to the concurrent effect of the interparticle interactions [41] and the surface anisotropy [40]. Specifically, the decrease in size of the $\mathrm{Fe}_{3} \mathrm{O}_{4}$ nanoparticles results in an increased surface spin disorder due to a higher fraction of superficial iron ions with incomplete coordination environments and broken bonds [27]. In such conditions, the surface effects become dominant eventually leading to the increase of the anisotropy energy constant [42]. In figure 4 the hysteresis loops of the three samples of oleate-coated $\mathrm{Fe}_{3} \mathrm{O}_{4}$ nanocrystals measured at $300(a)$ and $5 \mathrm{~K}(b)$ are presented.

In agreement with the $\mathrm{ZFC} / \mathrm{FC}$ measurements, the $M(H)$ curves recorded at $300 \mathrm{~K}$ confirm that the 6.6 and $11.6 \mathrm{~nm}$ $\mathrm{Fe}_{3} \mathrm{O}_{4}$ particles present a superparamagnetic behaviour, whereas those with a size of $17.8 \mathrm{~nm}$ are ferromagnetic exhibiting very low remanence $\left(M_{\mathrm{R}}\right)$ and coercivity $\left(H_{\mathrm{C}}\right)$. Although due to the limitations imposed by the experimental conditions a much more precise tuning of the particle size and, furthermore, an exact determination of the maximum $T_{\mathrm{B}}$ for which superparamagnetic relaxation is observed were not possible, these results clearly suggest that superparamagnetism in the $\mathrm{Fe}_{3} \mathrm{O}_{4}$ nanocrystals synthesized in diethylene glycol solutions occur in the size range of $12-18 \mathrm{~nm}$. Additionally, the reported critical size is in good agreement with the value of $20 \mathrm{~nm}$, a value often proposed as the superparamagnetic limit for the $\mathrm{Fe}_{3} \mathrm{O}_{4}$ nanoparticles. The corresponding values of saturation magnetization $\left(M_{\mathrm{S}}\right)$, squareness ( $\left.\mathrm{SQ}=M_{\mathrm{R}} / M_{\mathrm{S}}\right)$ and coercivity $\left(H_{\mathrm{C}}\right)$ are summarized in table 1 .

In the superparamagnetic state, the $M(H)$ curves of the nanosized materials can be described by the Langevin function (equation (1)):

$$
\begin{aligned}
M(T) & =M_{\mathrm{S}} L(x)=M_{\mathrm{S}}\left[\operatorname{coth}(x)-\frac{1}{x}\right] \\
= & M_{\mathrm{S}}\left(\frac{e^{x}+e^{-x}}{e^{x}-e^{-x}}-\frac{1}{x}\right),
\end{aligned}
$$

where $M(T)$ is the magnetization of the nanosized material at the temperature $T, M_{\mathrm{S}}$ represents the saturation magnetization of the nanosized material, $x=\mu H / k_{\mathrm{B}} T$, and the magnetic moment of the nanoparticles $\mu=M_{\mathrm{S} \text { (bulk) }} V$ ( $V$ stands for
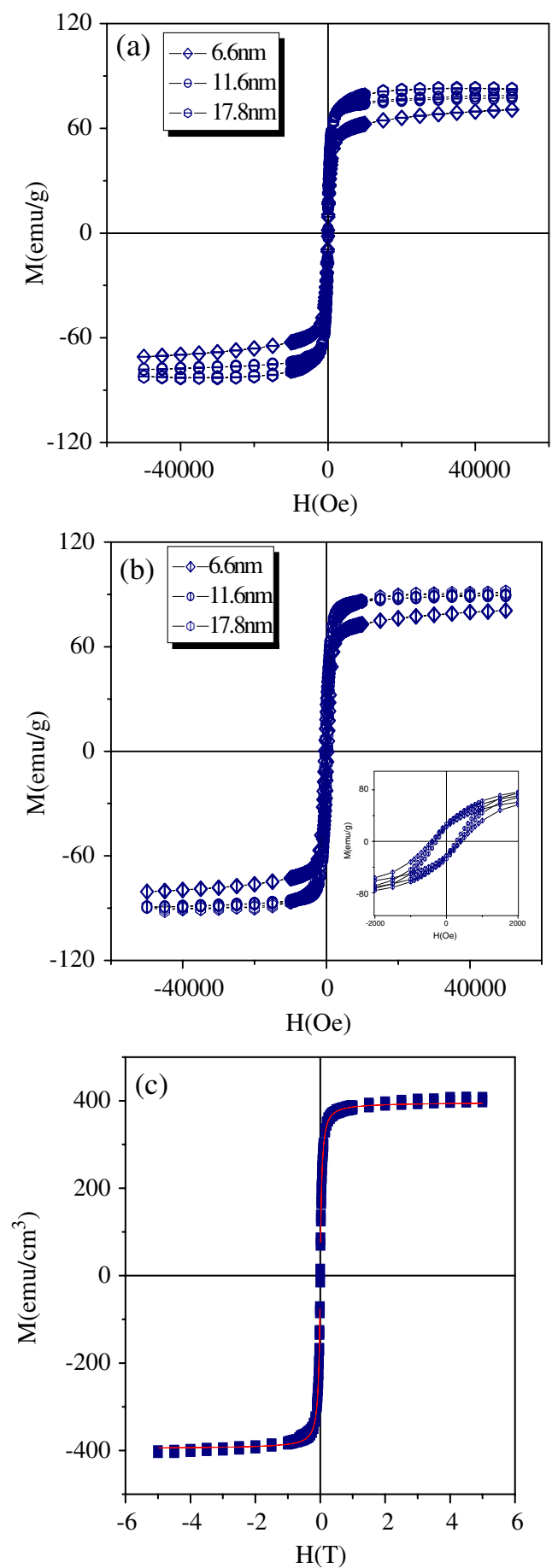

Figure 4. Magnetization $(M)$ as a function of the magnetic field $(H)$ measured at $300 \mathrm{k}(a)$ and $5 \mathrm{~K}(b)$ for oleate-capped magnetite nanocrystals with average sizes of $6.6 \mathrm{~nm}, 11.6 \mathrm{~nm}$ and $17.8 \mathrm{~nm}$, respectively; the fit of the $M$ versus $H$ curve was recorded at $300 \mathrm{~K}$ for $11.6 \mathrm{~nm}$ sized $\mathrm{Fe}_{3} \mathrm{O}_{4}$ nanoparticles $(c)$.

the average volume of the nanoparticles) [43]. The fit of the $M(H)$ curve for the $\mathrm{Fe}_{3} \mathrm{O}_{4}$ particles with the average diameter of $11.6 \mathrm{~nm}$ (as determined from the TEM measurement) is represented in figure $4(c)$, whereas the 'magnetic' sizes of the nanoparticles prepared from neat DEG and a 1:1 mixture of DEG and NMDEA are presented in table 1. The diameters of the oleate-capped $\mathrm{Fe}_{3} \mathrm{O}_{4}$ nanocrystals determined from the 
Table 1. Magnetic properties of variable-sized magnetite nanocrystals capped with oleate ligand.

\begin{tabular}{|c|c|c|c|c|c|c|c|c|}
\hline \multicolumn{2}{|c|}{$\begin{array}{l}\text { Average size } \\
\qquad(\mathrm{nm})\end{array}$} & \multirow[b]{2}{*}{$T_{\mathrm{B}}(\mathrm{K})$} & \multicolumn{2}{|c|}{$\begin{array}{c}M_{\mathrm{S}} \\
\left(\mathrm{emu} \mathrm{g}^{-1}\right)\end{array}$} & \multicolumn{2}{|c|}{$\begin{array}{l}H_{\mathrm{C}} \\
(\mathrm{Oe})\end{array}$} & \multicolumn{2}{|c|}{ SQ } \\
\hline TEM & Fit & & $5 \mathrm{~K}$ & $300 \mathrm{~K}$ & $5 \mathrm{~K}$ & $300 \mathrm{~K}$ & $5 \mathrm{~K}$ & $300 \mathrm{~K}$ \\
\hline 6.6 & 5.84 & 203 & 80.8 & 70.7 & 405.6 & 15.7 & 0.280 & 0.0240 \\
\hline 11.6 & 9.95 & 263.6 & 89.2 & 77.4 & 278.2 & 14.7 & 0.250 & 0.0312 \\
\hline 17.8 & - & $>300$ & 91.3 & 82.5 & 379.4 & 3.4 & 0.293 & 0.0076 \\
\hline
\end{tabular}

fitting of the $M$ versus $H$ curves are consistently smaller than those estimated from the TEM measurements.

Such a discrepancy was previously reported in the literature, being ascribed to the existence of a magnetically inert layer at the surface of the nanoparticles [44]. Due to their broken bonds, the iron ions contained in this superficial layer present a random orientation of their magnetic moments, thereby leading to a decrease of the total magnetization of each individual nanoparticle [38]. The data presented in table 1 show that the saturation magnetization $\left(M_{\mathrm{S}}\right)$ of the oleate-capped $\mathrm{Fe}_{3} \mathrm{O}_{4}$ nanocrystals increases continuously by increasing their average diameter, whereas the coercivity $\left(H_{\mathrm{C}}\right)$ follows an opposite trend. The magnetization values were obtained by the linear interpolation of the $M$ versus $1 / H$ variation at $5 \mathrm{~K}$ and $300 \mathrm{~K}$, respectively. The saturation magnetization at $300 \mathrm{~K}$ has values which are systematically lower than those measured at $5 \mathrm{~K}$ presumably due to thermal fluctuations of the magnetic moments of the nanoparticles at higher temperatures. At $300 \mathrm{~K}$, the saturation magnetization increases from $70.7 \mathrm{emu}^{-1}$ for the nanocrystals prepared from neat DEG $(6.6 \mathrm{~nm})$ to $82.5 \mathrm{emu}^{-1}$ for the nanoparticles obtained from neat NMDEA $(17.8 \mathrm{~nm})$. Nanocrystalline $\mathrm{Fe}_{3} \mathrm{O}_{4}$ particles synthesized from the $1: 1$ mixture of the two polyols $(11.6 \mathrm{~nm})$ have an intermediate value of $77.4 \mathrm{emu} \mathrm{g}^{-1}$ for the $M_{\mathrm{s}}$. These values are comparable to those of $\mathrm{Fe}_{3} \mathrm{O}_{4}$ nanoparticles having similar sizes obtained by other nonaqueous approaches. Specifically, while the $16 \mathrm{~nm}$ sized $\mathrm{Fe}_{3} \mathrm{O}_{4}$ particles prepared at $\sim 300^{\circ} \mathrm{C}$ by the 'seed-mediated growth' method possess a saturation magnetization of $83 \mathrm{emu} \mathrm{g}^{-1}$ [46], those with a diameter of $11 \mathrm{~nm}$ obtained at $180^{\circ} \mathrm{C}$ by a solvothermal technique in ethylene glycol medium have a $M_{\mathrm{s}}$ value of $79.2 \mathrm{emu} \mathrm{g}^{-1}$ [47]. It is worth noting that for the particles prepared in DEG solutions $(6.6 \mathrm{~nm})$, the saturation magnetization is much higher than $49 \mathrm{emu} \mathrm{g}^{-1}$, the $M_{\mathrm{s}}$ value measured experimentally for $7 \mathrm{~nm} \mathrm{Fe}_{3} \mathrm{O}_{4}$ particles synthesized at $88^{\circ} \mathrm{C}$ in an aqueous solution by the coprecipitation of the iron salts [48]. The decrease of the saturation magnetization $\left(M_{\mathrm{S}}\right)$ with the reduction of the nanocrystal size is associated with the existence of a surface layer where the iron ions possess unsaturated coordination spheres. This is due to the absence of some oxygen ions from the spinel lattice and/or the capping of the nanoparticles with long-chain carboxylate ligands which are bound directly to the iron ions. Such structural modifications create a surface spin disorder which induces significant changes in the magnetic properties of the nanocrystalline materials $[27,39,45,49,50]$. It was demonstrated that the nature of the capping ligand can also play an important role in the magnetic properties of the nanoparticles. For example, Gao and coworkers showed that for $\mathrm{Fe}_{3} \mathrm{O}_{4}$ particles with similar sizes $(8 \mathrm{~nm})$ prepared by the

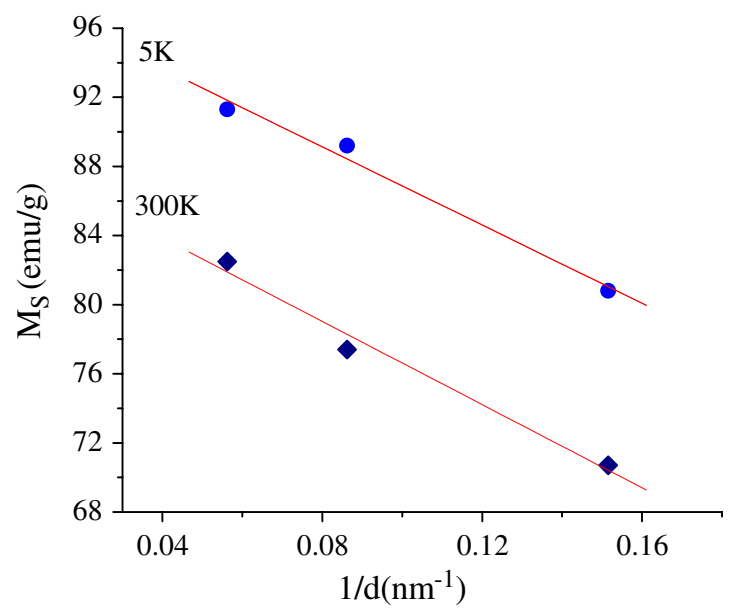

Figure 5. Saturation magnetization $\left(M_{\mathrm{S}}\right)$ versus the reciprocal of the average diameter $(1 / d)$ of the oleate-capped $\mathrm{Fe}_{3} \mathrm{O}_{4}$ nanoparticles.

solvothermal method, $M_{\mathrm{s}}$ increases from 89.8 to $96.6 \mathrm{emu} \mathrm{g}_{\mathrm{Fe}}^{-1}$ when tri- $n$-octylphosphine oxide (TOPO) is used as a capping ligand instead of hexadecylamine (HDA). Such an increase of the $M_{\mathrm{S}}$ value was attributed to the $\pi$-acceptor properties of the tri- $n$-octylphosphine oxide. Unlike the hexadecylamine, the TOPO molecules accept electrons from the iron ions once attached to the nanocrystals' surface, thus altering the exchange interactions between the neighbouring iron ions and eventually leading to the decrease of the saturation magnetization of the $\mathrm{Fe}_{3} \mathrm{O}_{4}$ nanoparticles [47].

Assuming that each nanoparticle consists of a magnetic core having the magnetic structure of the bulk material and a magnetically disordered shell, Chen and coworkers calculated the thickness of the shell $(t)$ from the variation of the saturation magnetization $\left(M_{\mathrm{S}}\right)$ with the reciprocal of the average diameter $(1 / d)$ of the particles [51]:

$$
M_{\mathrm{S}}=M_{S_{\text {(bulk })}}\left(1-\frac{6 t}{d}\right) .
$$

The linear fitting of our data obtained at $300 \mathrm{~K}$ (figure 5) yielded a value for the saturation magnetization of the bulk material $\left(M_{\mathrm{S} \text { (bulk) }}\right)$ of $88.65 \mathrm{emu}^{-1}$ and a thickness of the magnetically inert layer $(t)$ of $2.26 \AA$, whereas at $5 \mathrm{~K}$ the calculated values were $98.18 \mathrm{emu} \mathrm{g}^{-1}\left(M_{\mathrm{S}(\text { bulk })}\right)$ and $1.92 \AA(t)$, respectively. The $t$ values obtained from our calculations for oleate-capped $\mathrm{Fe}_{3} \mathrm{O}_{4}$ nanocrystals are much smaller than those of $4.5 \AA$ (at $20 \mathrm{~K}$ ) [29], $5 \AA$ (at $5 \mathrm{~K}$ ) [51] and $6 \AA$ (at $300 \mathrm{~K}$ ) [52] reported in the literature for nanosized $\mathrm{MnFe}_{2} \mathrm{O}_{4}$ particles prepared by other wet chemical methods (the w/o microemulsion method and coprecipitation in aqueous solutions) or $10 \AA$ (at $5 \mathrm{~K}$ ) [45] observed in the case of $\mathrm{CoFe}_{2} \mathrm{O}_{4}$ nanoparticles synthesized by coprecipitation in an aqueous solution followed by calcination.

Additionally, the coercivity was found to decrease with increasing volume of the $\mathrm{Fe}_{3} \mathrm{O}_{4}$ nanoparticles. Such a variation is in good agreement with Stoner-Wolhfarth theory for single-domain particles with uniaxial anisotropy, which predicts that the coercivity $\left(H_{\mathrm{C}}\right)$ of the nanosized materials depends on both the anisotropy constant $(K)$ and the saturation magnetization $\left(M_{\mathrm{S}}\right)$ :

$$
H_{\mathrm{C}}=\frac{2 K}{\mu_{0} M_{\mathrm{S}}},
$$


Magnetic properties of variable-sized $\mathrm{Fe}_{3} \mathrm{O}_{4}$ nanoparticles

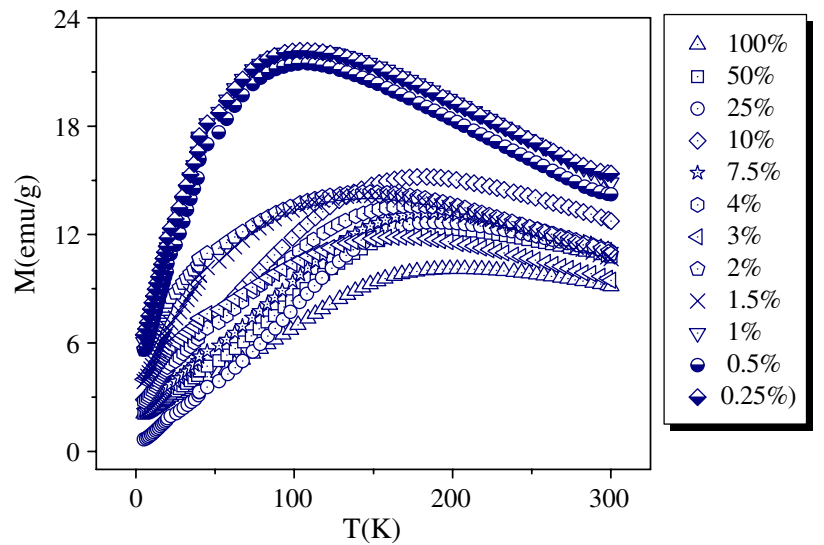

Figure 6. Typical ZFC curves of the $6.6 \mathrm{~nm}$ sized $\mathrm{Fe}_{3} \mathrm{O}_{4}$ particles capped with oleate ligand and diluted in paraffin; the concentration of the nanoparticles in the magnetite-paraffin solid solution varies between 0.25 and $100 \mathrm{wt} \%$.

where $\mu_{0}$ is the permeability constant of the vacuum [53,54]. Thus, the decrease of the coercivity with increasing size of the nanoparticles is consistent with both the decrease of the anisotropy constant and the increase of the saturation magnetization. The inset of figure $4(b)$ shows the hysteresis loops of the variable-sized $\mathrm{Fe}_{3} \mathrm{O}_{4}$ nanoparticles recorded at $5 \mathrm{~K}$. In all the cases the samples present a ferromagnetic behaviour with the saturation magnetization $\left(M_{\mathrm{S}}\right)$ ranging from 80.8 to $91.3 \mathrm{emu} \mathrm{g}^{-1}$ and coercivities varying between 278.2 and 405.6 Oe. The values of the reduced remanence or squarness ( $\mathrm{SQ}=M_{\mathrm{R}} / M_{\mathrm{S}}$ ) calculated from the $M(H)$ loops at $5 \mathrm{~K}$ range between 0.25 and 0.29 . They are considerable smaller than the theoretical value $\mathrm{SQ}=0.5$ suggesting that the oleate-capped $\mathrm{Fe}_{3} \mathrm{O}_{4}$ nanoparticles are single-domain and possess a uniaxial anisotropy [55].

In order to demonstrate the influence of the dipolar interparticle interactions on the magnetic properties of the oleate-capped $\mathrm{Fe}_{3} \mathrm{O}_{4}$ nanoparticles, dc magnetization measurements were performed on samples consisting of particles prepared from neat DEG $(6.6 \mathrm{~nm})$ and dispersed in paraffin. The concentration of the nanosized particles in the magnetite-paraffin solid solution was varied in a wide range (from 0.25 to $100 \mathrm{wt} \%$ ), thus allowing us to tune the interparticle distances. In figure 6 the typical temperature dependence magnetization curves measured in the ZFC mode under a static magnetic field of $100 \mathrm{Oe}$ for different concentrations of the $6.6 \mathrm{~nm} \mathrm{Fe}_{3} \mathrm{O}_{4}$ particles in the paraffin matrix are plotted. As a general feature, the magnetization decreases with increasing concentration of the nanosized particles. For example, the highest magnetization $\left(M=22.2 \mathrm{emu}^{-1}\right)$ corresponds to the most diluted sample ( $c=0.25 \%$ ), whereas the lowest value of magnetization ( $\left.M=10.1 \mathrm{emu} \mathrm{g}^{-1}\right)$ was obtained for the sample containing bare $\mathrm{Fe}_{3} \mathrm{O}_{4}$ nanoparticles $(c=100 \%)$. A similar trend was reported by El-Hilo et al in the case of $10 \mathrm{~nm} \mathrm{Fe}_{3} \mathrm{O}_{4}$ particles obtained by coprecipitation in an aqueous medium and suspended in a liquid carrier [56]. However, for the intermediate concentrations, the magnetization does not increase regularly with the dilution, the results being analogous to those obtained by Vestal et al for $8 \mathrm{~nm}$ sized $\mathrm{MnFe}_{2} \mathrm{O}_{4}$ particles synthesized by the 'seed-mediated growth' method
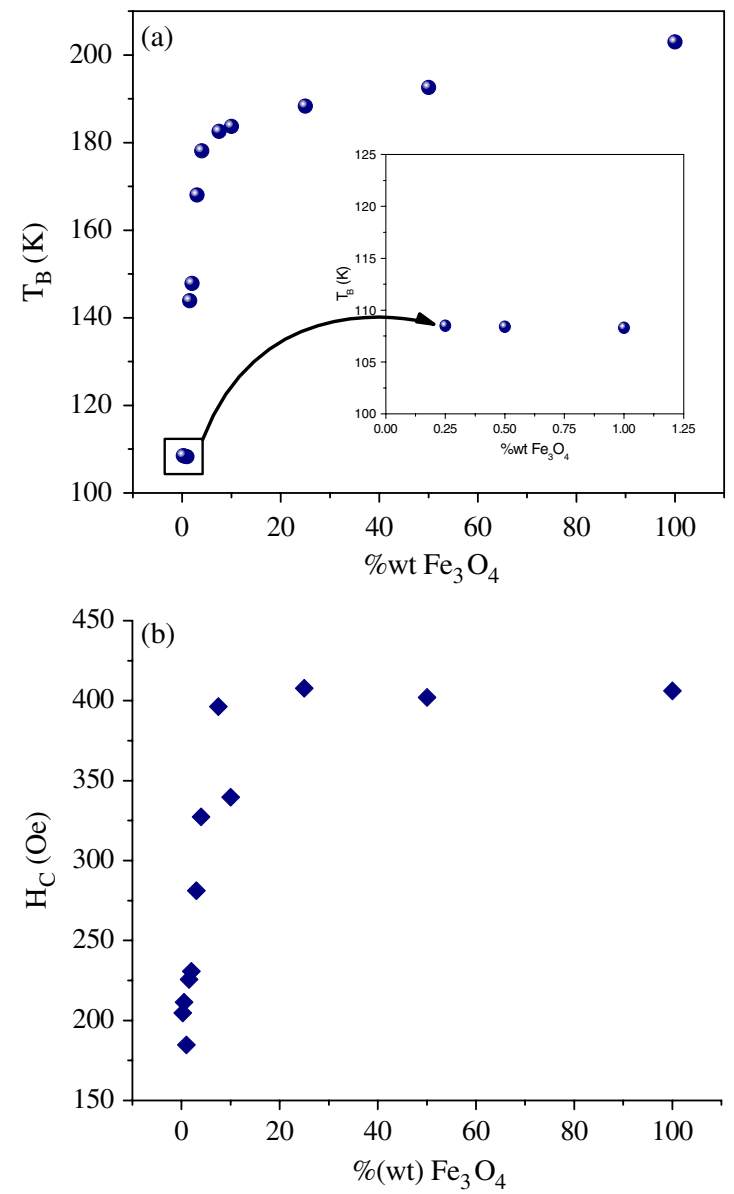

Figure 7. Variation of the blocking temperature $(a)$ and coercivity $(b)$ with the concentration of the $6.6 \mathrm{~nm}$ sized $\mathrm{Fe}_{3} \mathrm{O}_{4}$ particles in the paraffin matrix.

and dispersed in eicosane [57]. Although the origin of these discrepancies was not completely elucidated, the variation of the magnetization with the dilution strongly suggests that the interactions between the nanoparticles play an important role in modifying the anisotropy energy barriers of the magnetic system.

In figures $7(a)$ and $(b)$ the variations of the blocking temperature $\left(T_{\mathrm{B}}\right)$ and coercivity $\left(H_{\mathrm{C}}\right)$ with the dilution in paraffin are displayed. As seen in figure $7(a), T_{\mathrm{B}}$ decreases slightly from 203 to $183 \mathrm{~K}$ as the particle concentration is decreased from $100 \%$ to $7 \%$ and then drops sharply to a value of $108 \mathrm{~K}$ as the concentration reaches $1 \%$. After this abrupt decrease, further reduction of the particle concentration to $0.25 \%$ does not change the value of the blocking temperature. Likewise, the coercivity decreases from 409 to 397 Oe in the same concentration range and then drops abruptly to $185 \mathrm{Oe}$ for a concentration of $0.25 \%$ (figure $7(b)$ ). Such similarities in the variation pattern of both the blocking temperature and the coercivity with the particle concentration suggest that they have the same origin, that is, the interactions between nanoparticles.

This can be explained by the Dormann-Bessais-Fiorani (DBF) model which introduces an extra energy factor $\left(B_{\mathrm{i}}\right)$ in the expression of the anisotropy energy barrier $\left(E_{\mathrm{A}}\right)$ of a 
magnetic system with interparticle interactions (equation (3)):

$$
E_{\mathrm{A}}=K V \sin ^{2} \theta+B_{\mathrm{i}},
$$

where $K$ represents the anisotropy constant, $V$ is the volume of the magnetic particle, $\theta$ stands for the angle between the easy axis of the magnetic particle and the magnetization direction in an applied magnetic field and $B_{\mathrm{i}}$ is an energy factor describing the interparticle interactions [58,59]. Usually, the magnetostatic interactions between the particles include the exchange and the dipole-dipole interactions. However, when the nanocrystals' surface is functionalized with longchain organic molecules the exchange couplings are minimized and, thus, the energy factor $B_{\mathrm{i}}$ from the expression of the anisotropy energy barrier $\left(E_{\mathrm{A}}\right)$ is dominated by the dipolar interactions. For a magnetic system of single-domain particles, the energy corresponding to the dipole-dipole interactions can be expressed as:

$$
E_{\text {dipole-dipole }}=-\frac{\mu_{0} m_{0}^{2}}{4 \pi d^{3}}
$$

where $\mu_{0}$ represents the permeability constant of the vacuum and $m_{0}$ stands for the magnetic moment of the particle [51]. According to the above equation, the increase of the interparticle separation $(d)$ induces the reduction of the strength of the dipolar interactions leading, eventually, to the decrease of the anisotropy energy barrier $\left(E_{\mathrm{A}}\right)$. Under these conditions, the decrease of the blocking temperature and the coercivity with increasing interparticle separation can be explained by the lowering of the anisotropy energy barriers when the concentration of the oleate-capped $\mathrm{Fe}_{3} \mathrm{O}_{4}$ particles in the paraffin matrix is reduced. Below a critical concentration $(c=1 \%)$, the interparticle interactions become extremely weak so that a further decrease of the concentration to $0.25 \%$ will induce no variation in the value of the blocking temperature. The $M(H)$ curves of a sample containing $6.6 \mathrm{~nm}$ oleate-capped $\mathrm{Fe}_{3} \mathrm{O}_{4}$ particles dispersed in paraffin $(c=1 \%)$ were measured at different temperatures ranging from 10 to $300 \mathrm{~K}$ (figure $8(a)$ ) and the values of the saturation magnetization $\left(M_{\mathrm{S}}\right)$ were determined. The variation of the high-field magnetization with the temperature for a ferro- or ferrimagnetic system can be described by the Bloch law:

$$
M_{\mathrm{S}}(T)=M_{\mathrm{S}}(0)\left(1-B T^{\alpha}\right),
$$

where $M_{\mathrm{S}}(0)$ is the saturation magnetization at $0 \mathrm{~K}, B$ represents the Bloch constant and $\alpha$ stands for the Bloch exponent $[60,61]$. The values of $M_{\mathrm{S}}(0), B$ and $\alpha$ obtained by fitting the experimental data to the above equation (figure $8(b)$ ) are $93.5 \mathrm{emu} \mathrm{g}^{-1}, 10^{-5} \mathrm{~K}^{-1.67}$ and 1.67 , respectively.

As a feature of the oleate-capped $\mathrm{Fe}_{3} \mathrm{O}_{4}$ nanoparticles, the Bloch exponent in the fitting equation is slightly larger than the value of 1.5 characteristic to the bulk crystalline material [62] and which was previously reported for $3.3 \mathrm{~nm}$ $\mathrm{CoFe}_{2} \mathrm{O}_{4}$ nanoparticles obtained by the same technique [43]. Such a deviation from the Bloch law was observed for various nanoparticulate oxides, where the exponent $\alpha$ increases with reducing size of the nanoparticles [51,63]. Furthermore, the wide range of values of the Bloch exponent was ascribed to the influence of various factors such as the high surface/volume ratio, the preparative route, the chemical composition of the
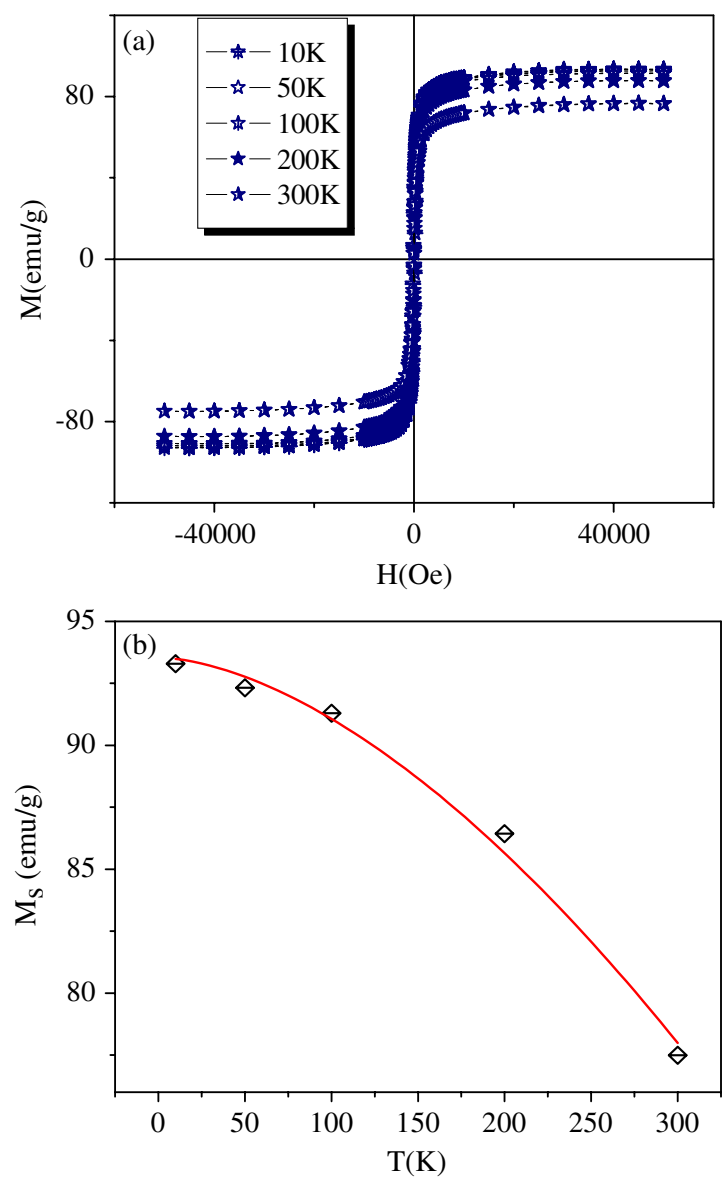

Figure 8. $\mathrm{M}(\mathrm{H})$ curves of the $6.6 \mathrm{~nm}$-sized $\mathrm{Fe}_{3} \mathrm{O}_{4}$ particles dispersed in paraffin $(c=1 \%)$ measured at different temperatures ranging from 10 to $300 \mathrm{~K}(a)$; the temperature dependence of the saturation magnetization

nanoparticles, as well as their surface modification [62]. For example, Chen et al suggested that in the case of the $\mathrm{MnFe}_{2} \mathrm{O}_{4}$ nanocrystals with sizes varying between 5 and $15 \mathrm{~nm}$ the values of the Bloch exponent fall within the range 1.6-2, whereas a value of 1.66 was reported for $12 \mathrm{~nm} \mathrm{La}_{0.8} \mathrm{Sr}_{0.2} \mathrm{MnO}_{3-\delta}$ nanoparticles obtained by a ball-milling process, which is substantially larger than $\alpha=0.9$ calculated by Xiong and coworkers for $8.1 \mathrm{~nm} \mathrm{CoCrFeO}_{4}$ nanoparticles prepared by a sol-gel approach $[51,63,64]$.

\section{Conclusions}

Variable-sized $\mathrm{Fe}_{3} \mathrm{O}_{4}$ nanoparticles were prepared in nonaqueous solutions of polyols and their magnetic properties were investigated. The average particle size estimated from the magnetization measurement in the superparamagnetic state is in good agreement with the TEM data. Both finite size and interparticle interaction effects were identified to influence the magnetic behaviour of the oleate-capped nanosized particles. With increasing size of the nanoparticles the anisotropy energy barriers increase and superparamagnetic behaviour is replaced by ferromagnetism. Similarly, the increase of the interparticle distances leads to a higher anisotropy which, in turn, leads to a substantial increase of the coercivity and the blocking temperatures. 


\section{Acknowledgments}

This work was supported by the DARPA through Grant No HR0011-04-C-0068. The authors would like to thank Dr Vicky Colvin and all members of her research group for the invaluable help in accommodating them and providing support in the aftermath of Hurricane Katrina.

\section{References}

[1] West A R 1984 Basic Solid State Chemistry (New York: Wiley) p 57

[2] Smart L and Moore E 1992 Solid State Chemistry: An Introduction (Singapore: Fong and Sons Printers Pte Ltd) p 24

[3] Kim J K, Lee J H and Lee S H 2004 J. Magn. Magn. Mater. 279173

[4] Regan B O and Gratzel M 1991 Nature 353737

[5] Makovec D, Košak A and Drofenik M 2004 Nanotechnology $15 \mathrm{~S} 160$

[6] McMichael R D, Shull R D, Swaertzendruber L J and Bennett L H 1992 J. Magn. Magn. Mater. 11129

[7] Shen L, Laibinis P E and Alan Hatton T 1999 Langmuir 15447

[8] Chou S Y, Wei M S, Kraus P R and Fischer P B 1994 J. Appl. Phys. 766673

[9] Raj K and Moskowitz R 1990 J. Magn. Magn. Mater. 85233

[10] Si S, Li C, Wang X, Yu D, Peng Q and Li Y 2005 Cryst. Growth Des. 5391

[11] Koneracka M, Kopcansky P, Timko M, Ramchand C N, de Sequeira A and Trevan M 2002 J. Mol. Catal. B: Enzym. 1813

[12] Ferreira H A, Graham D L, Freitas P P and Cabral J M S 2003 J. Appl. Phys. 937281

[13] Cao Y C, Jin R, Nam J, Thaxton C S and Mirkin C A 2003 J. Am. Chem. Soc. 12514676

[14] Manent J, Oguievetskaia K, Bayer J, Ratner N and Giovannini M 2003 J. Neurosci. Methods 123167

[15] Doyle P S, Bibette J, Bancaud A and Viovy J L 2002 Science 2952237

[16] Kim D K, Mikhaylova M, Wang F H, Kehr J, Bjelke B, Zhang Y, Tsakalakos T and Mamoun M 2003 Chem. Mater. 154343

[17] Babes L, Denizot B, Tanguy G, Le Jeune J J and Jalet P 1999 J. Colloid Interface Science 212474

[18] Papisov M I, Bogdanov A Jr, Schaffer B, Nossiff N, Shen T, Weissleder R and Brady T J 1993 J. Magn. Magn. Mater. 122383

[19] Jordan A, Scholtz R, Wust P, Schbirra H, Schiesel T, Schmidt H and Felix R 1999 J. Magn. Magn. Mater. 194185

[20] Wunderbaldinger P, Josephson L and Weissleder R 2002 Bioconjugate Chem. 13264

[21] Gupta P K, Hung C T, Lam F C and Perrier D G 1988 Int. J. Pharm. $\mathbf{4 3} 167$

[22] Alexiou C, Arnold W, Klein R J, Parak F G, Hulin P, Bergemann C, Erhardt W, Wagenpfeil S and Lubbe A S 2000 Cancer Res. 606641

[23] Jain T K, Morales M A, Sahoo K S, Leslie-Pelecky D L and Labhasetwar V 2005 Molec. Pharm. 2194

[24] Ibrahim A, Couvreur P, Roland M and Speiser P 1982 J. Pharm. Pharmacol. 3559

[25] Awschalom D D and DiVencenzo D P 1995 Phys. Today 4843

[26] Si S, Kotal A, Mandal T K, Giri S, Nakamura H and Kohara T 2004 Chem. Mater. 163489

[27] Kodama R H, Berkowitz A E, McNiff E J and Foner S 1997 J. Appl. Phys. 815552

[28] Morales M P, Veintemillas-Verdaguer S, Montero M I, Serna C J, Roig A, Casas Ll, Martínez B and Sandiumenge F 1999 Chem. Mater. 113058
[29] Liu C and Zhang Z J 2001 Chem. Mater. 132092

[30] Sorensen C M 2002 Nanoscale Materials in Chemistry ed K J Klabunde (New York: Wiley) p 169

[31] Caruntu D, Remond Y, Chou N H, Jun M J, Caruntu G, He J, Goloverda G, O’Connor C J and Kolesnichenko V 2002 Inorg. Chem. 416137

[32] Caruntu D, Caruntu G, Chen Y, Goloverda G, O'Connor C J and Kolesnichenko V 2004 Chem. Mater. 165527

[33] Djurberg C, Svedlindh P, Nordblad P, Hansen M F, Bødker F and Mørup S 1997 Phys. Rev. Lett. 795154

[34] Jonsson T, Nordblad P and Svedlindh P 1998 Phys. Rev. B 57497

[35] del Barco E, Asenjo J, Zhang X X, Pieczynski R, Julìa A, Tejada J, Ziolo R F, Fiorani D and Testa A M 2001 Chem. Mater. 131487

[36] Suber L, Imperatori P, Ausanio G, Fabbri F and Hofmeister H 2005 J. Phys. Chem. B 1097103

[37] Ma M, Wu Y, Zhou J, Sun Y, Zhang Y and Gu N 2004 J. Magn. Magn. Mater. 26833

[38] Morup S and Topsoe H J 1976 Appl. Phys. 1163

[39] Lee Y, Lee J, Bae C J, Park J-G, Noh H-J, Park J-H and Hyeon T 2005 Adv. Funct. Mater. 15503

[40] Tung L D, Kolesnichenko V, Caruntu G, Caruntu D, Remond Y, Golub V O, O'Connor C J and Spinu L 2002 Physica B 319116

[41] Yokoyama M, Ohta E, Sato T and Sato T 1998 J. Magn. Magn. Mater. 183173

[42] Park J, An K, Hwang Y, Park J-G, Noh H-J, Kim J-Y, Park J-H, Hwang N-M and Hyeon T 2004 Nature Mater. 3891

[43] Charles S W and Popplewell J 1982 Ferromagnetic Materials vol 2 ed H Wohlfarth (Amsterdam: North-Holland)

[44] Kommareddi N S, Tata M, John V T, McPherson G L, Lee Y-S, O'Connor C J, Akkara J A and Kaplan D L 1996 Chem. Mater. 8801

[45] Grigorova M, Blythe H J, Blaskov V, Rusanov V, Petkov V, Masheva V, Nihtianova D, Martinez LI M, Muñoz J S and Mikhov M 1998 J. Magn. Magn. Mater. 183163

[46] Sun S, Zeng H, Robinson D B, Raoux S, Rice P M, Wang S X and Li G 2004 J. Am. Chem. Soc. 126273

[47] Hou Y, Yu J and Gao S 2003 J. Mater. Chem. 131983

[48] Gee S H, Hong Y K, Erickson D W, Park M H and Sur J C 2003 J. Appl. Phys. 937560

[49] Vestal C R and Zhang Z J 2004 Int. J. Nanotechnol. 1240

[50] Tung L D, Kolesnichenko V, Caruntu D, Chou N H, O'Connor C and Spinu J L 2003 J. Appl. Phys. 937486

[51] Chen J P, Sorensen C M, Klabunde K J, Hadjipanayis G C, Devlin E and Kostikas A 1996 Phys. Rev. B 549288

[52] Zheng M, Wu X C, Zou B S and Wang Y J 1998 J. Magn Magn. Mater. 183152

[53] McCurrie R A 1994 Ferromagnetic Materials-Structure and Properties (London: Academic) p 16

[54] Vestal C R and Zhang Z J 2002 Chem. Mater. 143817

[55] Stoner E C and Wohlfarth E P 1948 Phil. Trans. R. Soc. A 240 559

[56] El-Hilo M, O'Grady K and Chantrell R W 1992 J. Magn. Magn. Mater. 114295

[57] Vestal C R, Song Q and Zhang Z J 2004 J. Phys. Chem. B 10818222

[58] Frankamp B L, Boal A K, Tuominen M T and Rotello V M 2005 J. Am. Chem. Soc. 1279731

[59] Dormann J L, Fiorani D and Tronc E 1999 J. Magn. Magn. Mater. 202251

[60] Balzar D and Ledbetter H 1993 J. Appl. Crystallogr. 2697

[61] Bloch F 1931 Z. Phys. 61206

[62] Hochepied J F, Bonville P and Pileni M P 2000 J. Phys. Chem. B 104905

[63] Roy S, Dubenko I, Edorh D D and Ali N 2004 J. Appl. Phys. 961202

[64] Xiong G, Mai Z, Xu M and Cui S 2001 Chem. Mater. 131943 